\title{
NEONATAL CPAP TRANSFERS IN YORKSHIRE \& HUMBER - EMBRACE TRANSPORT SERVICE
}

\author{
S. Babarao ${ }^{1}$, C. Smith ${ }^{2}$, C. Harrison ${ }^{2}$, S. Hancock ${ }^{2}$ \\ ${ }^{I}$ Sheffield Children's Hospital, ${ }^{2}$ Transport, Sheffield Children's Hospital, Sheffield, UK
}

Background \& aims: From a central non-hospital base, the Embrace transport team co-ordinates paediatric \& neonatal transfers in Yorkshire \& Humber. It completes about 40 transports a week. Embrace transfers babies on CPAP support. Our aim was to review and evaluate our performance and adverse events during stabilisation \& transit with regards to neonatal CPAP transfers.

Methods: Retrospective audit of all neonatal CPAP transfers during the period April - August 2010

\section{Results:}

Number of neonatal transfers during the period: 603

Number of babies on CPAP at referral: 94

Number of babies transferred on CPAP: 51 ( $8.5 \%$ of total neonatal transfers)

Nurses and ANNPs led the transfer in 65\% (Back transfers) and middle grade doctors in $35 \%$ (Acute sick babies).

The stabilisation time was $<60$ minutes in 20 (39\%), 60-120 minutes in $45 \%$ and $>120$ minutes in $8(16 \%)$ of transfers. Apart from the transient increase in oxygen requirement and PEEP while on transport CPAP (50\% of transfers), the main adverse event was hypothermia (temperature $</=36.5{ }^{\circ} \mathrm{C}$ at destination hospital) in $33 \%$.

Lower the gestational age and the birth weight, greater was the risk of hypothermia. Stabilisation time and transfer time were negatively correlated with hypothermia.

Conclusions: Careful triaging of referrals led to efficient use of nursing teams in conducting safe CPAP transfers. Greater emphasis placed on temperature control has led to minimising the risk of hypothermia. A new cost-effective method to humidify gases through the CPAP circuit is currently on trial at Embrace. 